\title{
Identificación de incrustaciones en ciclones recuperadores de calor de hornos DOPOL
}

FER NANDo TRIVINo VAZQUEZ, Dr. en Ciencias Químicas IETce

\section{ANTECEDENTES}

En un ciclón recuperador de calor, situado a la entrada del horno de clinquerización tipo DOPOL, se producían unas incrustaciones sedimentarias que terminaban produciendo la obstrucción del ciclón.

Simultáneamente aparecían dificultades de marcha durante el crecimiento, por sedimentación-fusión, de dichas incrustaciones. Finalmente era necesaria la parada del horno para la limpieza del ciclón.

En una de las muestras obtenidas de la incrustación, en el difractograma realizado, se identificaron (1) los siguientes componentes: $\mathrm{CaCO}_{3}, \mathrm{CaO}, \mathrm{Ca}_{12} \mathrm{Al}_{14} \mathrm{O}_{33}, \mathrm{Ca}_{6} \mathrm{Al}_{4} \mathrm{Fe}_{2} \mathrm{O}_{15}$, $\mathrm{K}_{2} \mathrm{SO}_{4}$; en el difractograma quedaban sin identificar los siguientes espaciados:

\begin{tabular}{cc}
\hline Compuesto $\mathbf{H}$ & Fluorellestadita \\
\hline 5,00 & 4,07 \\
3,60 & 3,90 \\
3,48 & 3,46 \\
3,11 & 3,19 \\
2,83 & 2,82 \\
2,75 & 2,72 \\
2,66 & \\
2,18 & \\
2,14 & \\
1,98 & \\
1,79 & \\
\hline
\end{tabular}

En un estudio de los efectos del fluor y de los fosfatos en el sistema: $\mathrm{CaO}-\mathrm{SiO}_{2}-\mathrm{Al}_{2} \mathrm{O}_{3}$ $\mathrm{SO}_{3}$ realizado por SUDOH y NAKAMURA (3) los espaciados de la segunda columna eran comparados con los de la ellestadita por los autores; para simplificación llamaremos a continuación a dicho compuesto "fluorellestadita". Quedan sin identificar la columna de espaciados denominada, en lo sucesivo, "compuesto H".

La muestra calcinada en crisol de platino a $1.000^{\circ} \mathrm{C}$, para eliminar los carbonatos, tenía el siguiente análisis químico:

$$
\begin{array}{lll}
\mathrm{SiO}_{2}=16,6 \% & \mathrm{Fe}_{2} \mathrm{O}_{3}=3,1 \% & \mathrm{Cl}^{-}=0,8 \% \\
\mathrm{Al}_{2} \mathrm{O}_{3}=4,7 \% & \mathrm{MgO}=1,8 \% & \mathrm{~F}^{-}=1,5 \% \\
\mathrm{CaO}=50,8 \% & \mathrm{~K}_{2} \mathrm{O}=2,7 \% & \\
\mathrm{SO}_{3}=16,6 \% & \mathrm{Na}_{2} \mathrm{O}=0,2 \% &
\end{array}
$$




\section{INTENTO DE CONCENTRACION DEL COMPUESTO}

Dos gramos de la muestra calcinada y $100 \mathrm{ml}$ de etilenglicol se trataron a ebullición durante diez minutos, en un vaso de precipitados con un agitador magnético. El residuo se filtró, a través de un filtro rápido, y se lavó con alcohol metílico; seguidamente el filtro y residuo se calcinaron en un crisol de platino a $1.000^{\circ} \mathrm{C}$ durante cinco minutos.

El difractograma del residuo mostraba los picos correspondientes de los s.guientes compuestos: $\mathrm{CaSO}_{4}, \mathrm{CaF}_{2}, \mathrm{CaF}_{2} \cdot 3 \mathrm{Ca}_{3} \mathrm{SiSO}_{8}, \mathrm{Ca}_{12} \mathrm{Al}_{14} \mathrm{O}_{33}, \mathrm{y}$ "compuesto $\mathrm{H}$ ".

El residuo se trató con ácido salicílico-metanol (5) y quedó sin atacar el $70 \%$.

$\mathrm{El}$ difractograma de la parte no atacada mostraba los siguientes compuestos: $\mathrm{CaSO}_{4}$ $\mathrm{CaF}_{2} \cdot 3 \mathrm{Ca}_{3} \mathrm{SiSO}_{8}, \mathrm{Ca}_{12} \mathrm{Al}_{14} \mathrm{O}_{33}$ y trazas de $\mathrm{Ca}_{6} \mathrm{Al}_{4} \mathrm{Fe}_{2} \mathrm{O}_{15}$.

La parte soluble se desecó y calcinó en crisol de platino a $1.000^{\circ} \mathrm{C}$. El difractograma mostraba la presencia de beta silicato bicálcico.

Se hizo una mezcla de $0,7 \mathrm{~g}$ del residuo insoluble en salicílico-metanol y $0,3 \mathrm{~g}$ de beta silicato bicálcico (sintetizado a partir de gel de sílice y carbonato cálcico en relaciones estequiométricas) y se calentó en crisol de platino a $800^{\circ} \mathrm{C}$ durante 2 horas; el difractograma mostraba la presencia de los siguientes compuestos: $\mathrm{CaSO}_{4}, \beta-2 \mathrm{CaO} . \mathrm{SiO}_{2}, \mathrm{~K}_{2} \mathrm{SO}_{4} .2 \mathrm{CaSO}_{4}$, $\mathrm{Ca}_{12} \mathrm{Al}_{14} \mathrm{O}_{33}$ y como dudoso $\mathrm{CaF}_{2}$. Calentan to la mezcla en crisol de platino a $1.000^{\circ} \mathrm{C} \mathrm{du}-$ rante 5 días, en el difractograma correspondiənte se detectan los siguientes compuestos: $\mathrm{CaSO}_{4}, \mathrm{CaSO}_{4} \cdot 2\left(2 \mathrm{CaO} . \mathrm{SiO}_{2}\right), \mathrm{CaSO}_{4} \cdot 3\left(2 \mathrm{CaO} \mathrm{SiO}_{2}\right)$ y "compuesto $\mathrm{H}$ ".

De lo anterior se deduce que la fluorellestadita en presencia de alguno de los componentes, $\mathrm{MgO}, \mathrm{K}_{2} \mathrm{O}, \mathrm{Na}_{2} \mathrm{O}$ y Cl${ }^{-}$se descomponía formando el "compuesto $\mathrm{H}$ ".

Se hicieron sendas mezclas con cantidades proporcionales a 1 mol de fluorellestadita y 1 mol de $\mathrm{MgO}$, y también con $1 \mathrm{~mol}$ de $\mathrm{MgCl}_{2}$ se calentaron a $1.000^{\circ} \mathrm{C}$ durante 24 horas. En los difractogramas de dichas mezclas no se dətectó el "compuesto H". Se hizo otra nueva mezcla a la que se le añadió la cantidad proporcional de $\mathrm{NaOH}$ para obtener un mol expresado como $\mathrm{Na}_{2} \mathrm{O}$ y tampoco el difractograma manifestaba las líneas del "compuesto $\mathrm{H}$ ".

Al realizar el ensayo con $\mathrm{KOH}$ y fluorellestadita, sí se detectaron las líneas de difracción del "compuesto H" según se detalla a continuación.

\section{REACCIONES DEL $\mathrm{K}_{2} \mathrm{O}$ CON LA FLUORELLESTADITA}

Con el fin de averiguar la composición del "compueso H", se hicieron cuatro mezclas de $0,01 \mathrm{~mol}$ de fluorellestadita y respectivamente 0,$0025 ; 0,005 ; 0,01$ y 0,015 moles de $\mathrm{K}_{2} \mathrm{O}$, en forma de $\mathrm{KOH}$. Las mezclas se molieron en un molino de ágata durante $20 \mathrm{mi}-$ nutos y posteriormente, en un crisol de platino, se calentaron a una temperatura de $1.000^{\circ} \mathrm{C}$ durante 3 horas. Para cantidades tan pequeñas de $\mathrm{K}_{2} \mathrm{O}$ como 0,0025 moles, equivalentes a 0,25 moles de $\mathrm{K}_{2} \mathrm{O}$ con $1 \mathrm{~mol}$ de f́luorellestadita, se formaba el "compuesto $\mathrm{H}$ " -(figura 1-3, puro) junto con fluorellestadita (figura 1-1, pura) - según se puede observar en el correspondiente difractograma (figura 1-2). La formación máxima del "compuesto $\mathrm{H}$ " se observa para 0,005 moles de $\mathrm{K}_{2} \mathrm{O}$, es d icir, para la mezcla que contenía las relaciones molares $1 \mathrm{~mol}$ de fluorellestadita más $1 / 2 \mathrm{~mol}$ de $\mathrm{K}_{2} \mathrm{O}$ (figura 2-1). Para cantidades superiores de $\mathrm{K}_{2} \mathrm{O}$ correspondientes a 1 y 1,5 moles, primero se descompone toda la fluorellestadita (figura 2-2) y después también el "compuesto H" (figura 2-3). Para estas dos últimas mezclas en los difractogramas correspondientes se puede observar un aumento en la intensidad de las líneas correspondientes al: $\mathrm{CaO}, \beta-2 \mathrm{CaO} \mathrm{SiO}_{2}, \mathrm{~K}_{2} \mathrm{SO}_{4}$ y $\mathrm{CaF}_{2}$, siendo curiosa la desaparición del $\mathrm{CaSO}_{4}$.

Lo anterior nos indica que el "compuesto $\mathrm{H}$ " tiene como máx:mo los componentes de la fluorellestadita, es decir. $3\left(2 \mathrm{CaO} \mathrm{SiO}_{2}\right) ; 3 \mathrm{CaSO}_{4} ; \mathrm{CaF}_{2}$ (para este último, posiblemente me- 
RELLENO EN NEGRO:

RIPIENO IN NERO:

$(1,6 \leftrightarrow 2) \mathrm{CoO} \cdot \mathrm{KF} \cdot 3\left[2 \mathrm{CaO} \cdot \mathrm{SiO}_{2}\right] \cdot 2 \mathrm{CaSO}_{4}$

FILLED IN BLACK:

$\times \mathrm{CaF}_{2} \cdot 3 \mathrm{CaSiSO}_{8}$

- $\mathrm{K}_{2} \mathrm{SO}_{4}$

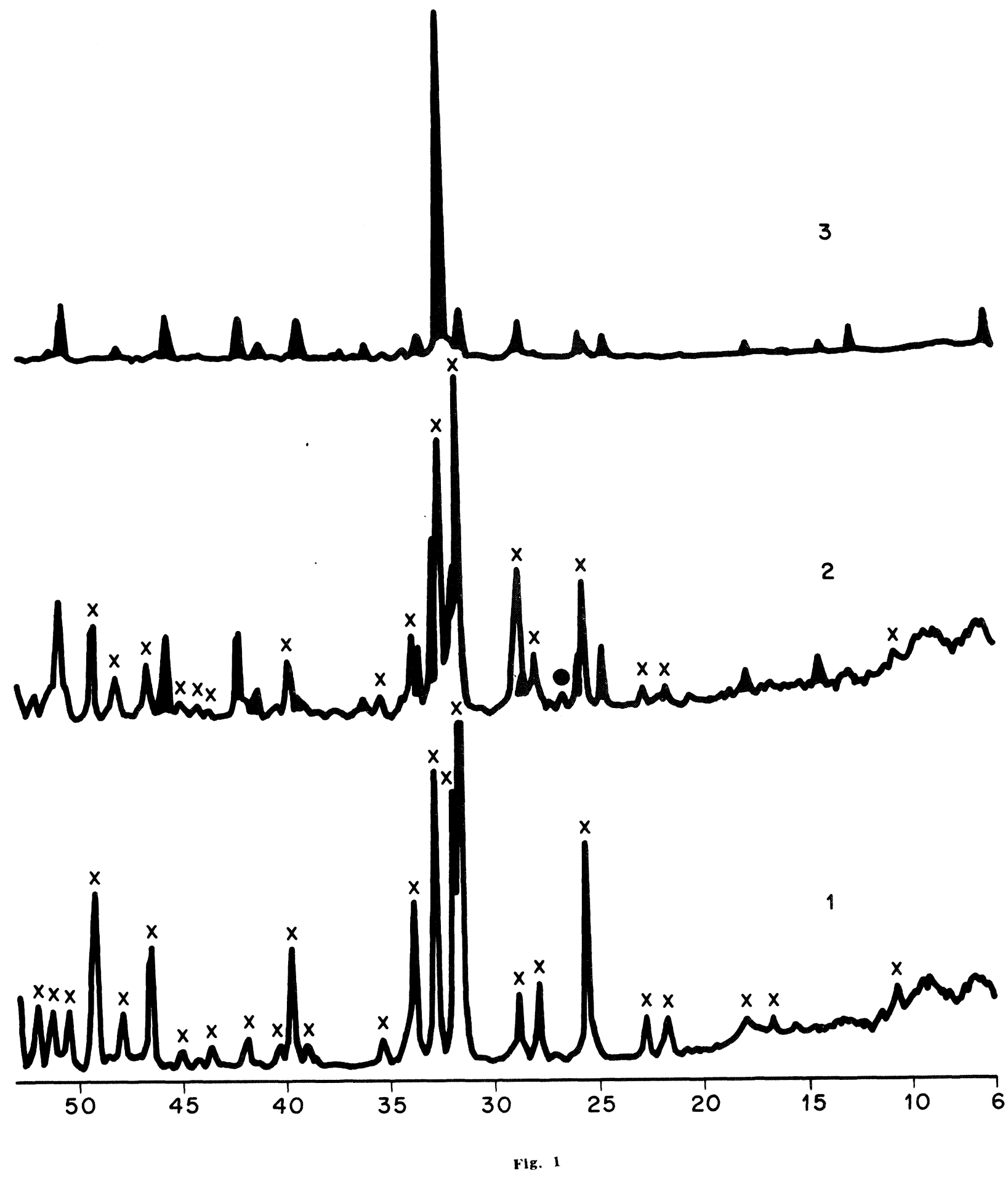



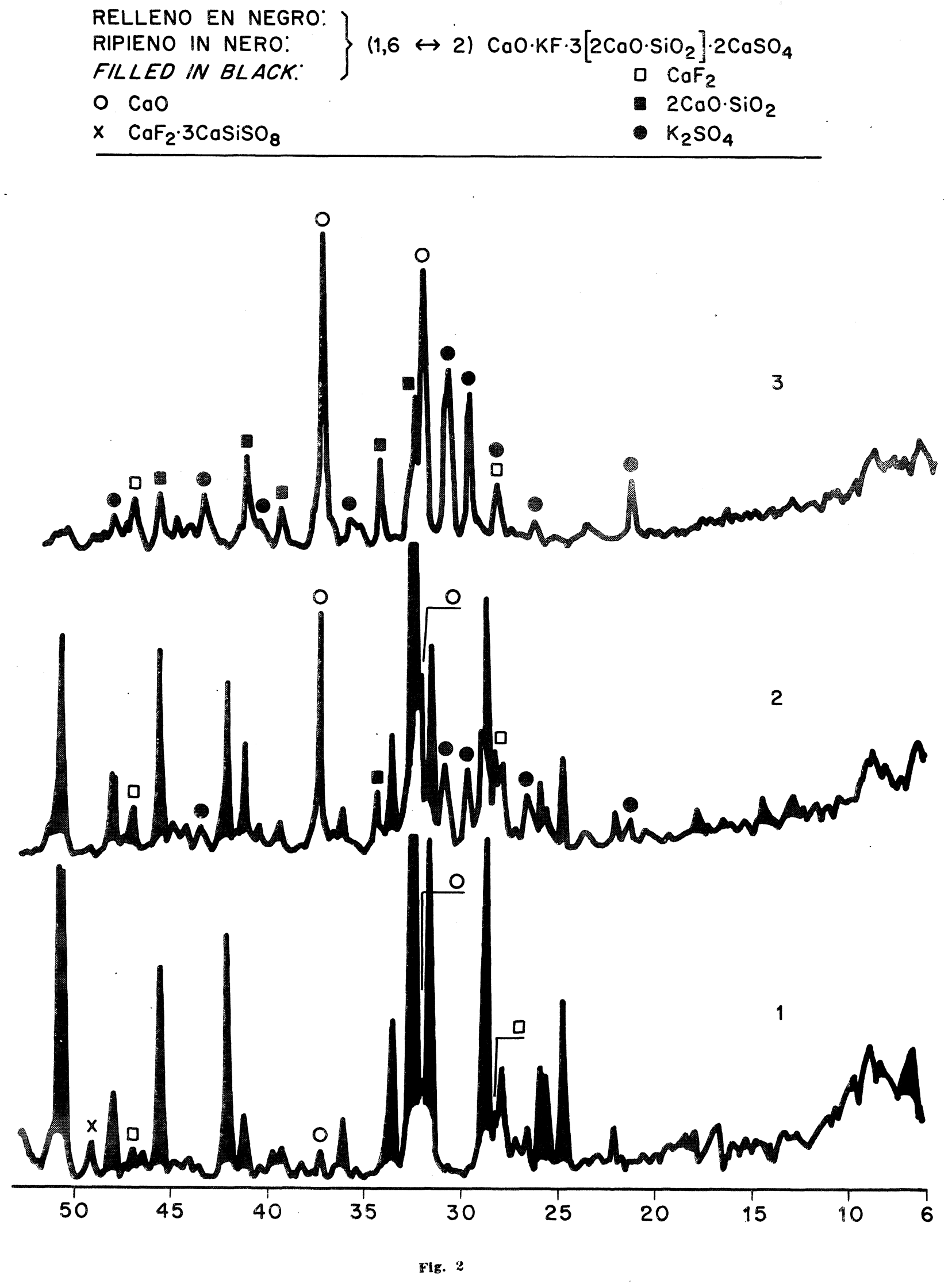


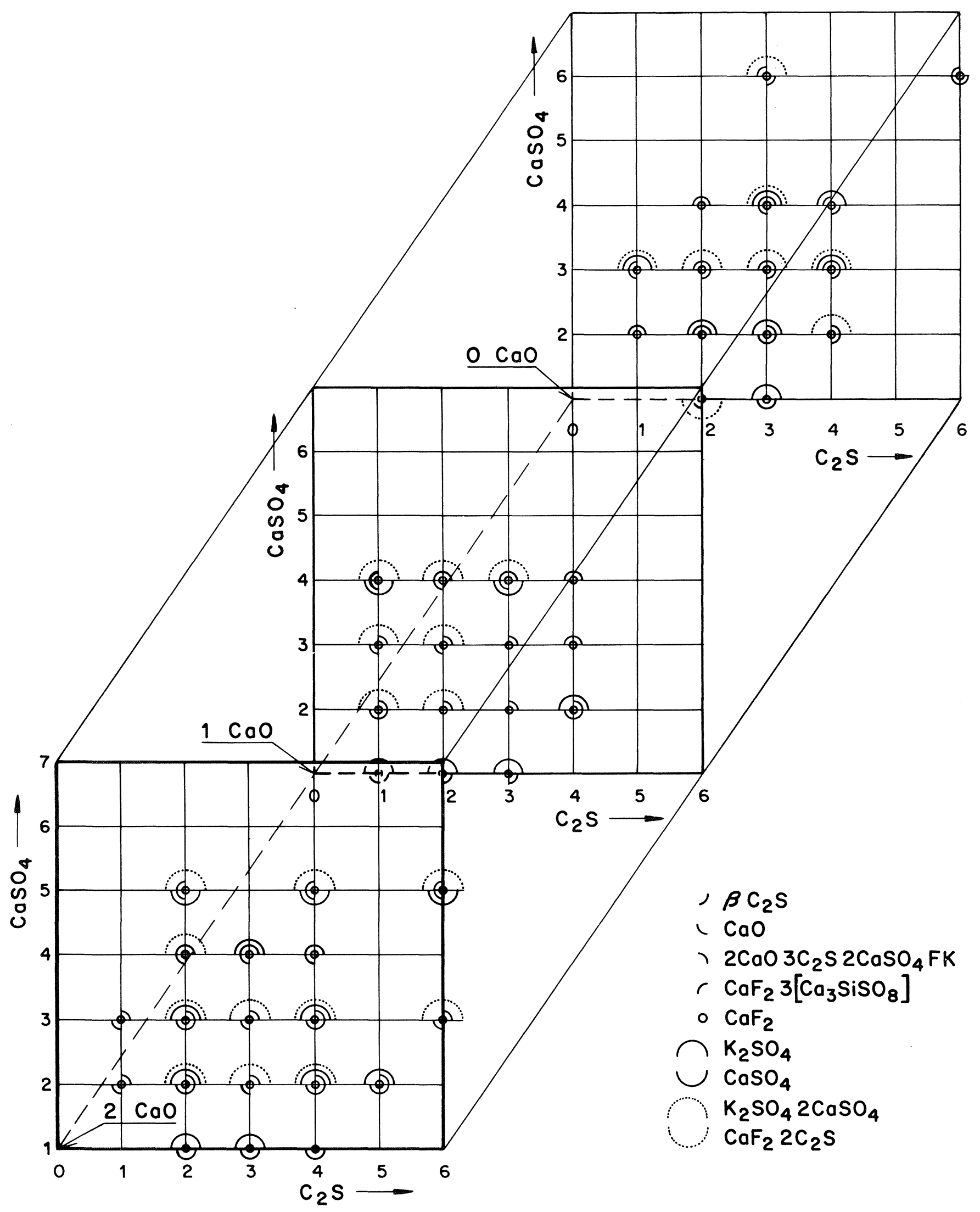

FIg. 3 
nos, véanse en el cuadro 1 las intensidades del $\mathrm{CaF}_{2}$ ) y para el $\mathrm{K}_{2} \mathrm{O} 1 / 2 \mathrm{~mol}$. Como la fluorellestadita tenía un exceso de $\mathrm{CaO}$ era muy posible también que el compuesto tuviera un exceso de $\mathrm{CaO}$ sobre el necesario para formar el silicato bicálcico (3).

\section{CUADRO 1}

\begin{tabular}{|c|c|c|c|c|c|c|}
\hline $\begin{array}{c}\text { Compuestos } \\
2 \theta= \\
= \\
\text { fluorellestadita } \\
+\mathbf{K}_{2} \mathbf{O} \text { moles } \\
\end{array}$ & 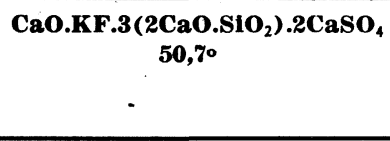 & $\begin{array}{l}\text { CaO } \\
\mathbf{3 7 , 4 ^ { \circ }}\end{array}$ & $\underset{477^{\circ}}{\mathbf{C a F}_{2}}$ & $\begin{array}{r}\mathbf{K}_{2} \mathrm{SO}_{4} \\
29,8^{\circ}\end{array}$ & $\underset{34,5^{\circ}}{2 \mathrm{CaO}^{\circ} \mathrm{SiO}_{2}}$ & $\begin{array}{l}\mathrm{CaF}_{2} \cdot \mathbf{3 C a} \mathrm{Ca}_{3} \mathrm{SiSO}_{8} \\
{ }_{49,2^{\circ}}\end{array}$ \\
\hline 1 & 0 & 15 & 0 & 0 & 0 & 85 \\
\hline $1+0,25$ & 50 & 0 & 4 & 0 & 0 & 45 \\
\hline $1+0,5$ & 85 & 8 & 10 & 0 & 0 & 10 \\
\hline $1+1$ & 50 & 65 & 12 & 22 & 16 & 0 \\
\hline $1+1,5$ & 0 & 100 & 17 & 40 & 24 & 0 \\
\hline
\end{tabular}

\section{SUBSISTEMA KF-CaSO $-2 \mathrm{CaO}_{4} \mathrm{SiO}_{2}-\mathrm{CaO}$}

En la figura 3 se pueden observar las mezclas realizadas a partir de las adecuadas proporciones molares de $\mathrm{K}_{2} \mathrm{SO}_{4}, \mathrm{CaF}_{2}, \mathrm{CaCO}_{3}, \mathrm{CaSO}_{4}$ y gel de sílice, molidas en molino de ágata durante 20 minutos y calentadas en crisol de platino a $1.000^{\circ} \mathrm{C}$ durante 3 horas. Los datos de la figura 3 se obtuvieron de los difractogramas correspondientes a las mezclas calcinadas. Para todas las mezclas se mantuvo constante la proporción de $2 \mathrm{KF}$ equivalente al $\mathrm{CaF}_{2}$ y como variables: para el eje $\mathrm{Y}$, el $\mathrm{CaSO}_{4}$; para el eje $\mathrm{X}$, el $2 \mathrm{CaO} \mathrm{SiO}_{2}$; y para el eje $\mathrm{Z}$, el $\mathrm{CaO}$. De los resultados indicados en la figura se pueden obtener las siguientes conclusiones:

1. La proporción de $1 \mathrm{~mol}$ de $\mathrm{CaF}_{2}<>2 \mathrm{KF}$ añadido es excesiva, ya que en todos los difractogramas de los diversos compuestos preparados se detectaban las líneas correspondientes al $\mathrm{CaF}_{2}$.

2. En las probables composiciones del "compuesto $H$ ", cuando la relación molar ensayada de $\mathrm{CaSO}_{4}$ era superior a 3, se identificaban en los difractogramas las líneas correspondientes a la fluorellestadita. Cuando no había $\mathrm{CaO}$ en exceso sucedía también para 3 moles de $\mathrm{CaSO}_{4}$. Si la relación era $1 \mathrm{CaSO}_{4}$ o menor en los difractogramas se identificaban las líneas correspondientes del $\beta-\mathrm{C}_{2} \mathrm{~S}^{*}$ ó $\mathrm{CaF}_{2} \cdot 2\left(2 \mathrm{CaO} \cdot \mathrm{SiO}_{2}\right)$.

3. La presencia en la composición de un exceso de $\mathrm{CaO}$ de 1 a 2 moles, sobre el necesario para formar $2 \mathrm{CaO} \mathrm{SiO}_{2}$, resulta imprescindible para eliminar la formación de la fluorellestadita y el $\beta-2 \mathrm{CaO} . \mathrm{SiO}_{2}$; normalmente en presencia de un exceso de $\mathrm{CaO}$ aparece sólo uno de los dos compuestos citados anteriormente o ninguno de ellos.

$\mathrm{Si}$ la $\mathrm{CaO}$ en exceso era de 2 moles, aparecía cal libre. No obstante se considera que con una duración del tratamiento mayor, temperaturas comprendidas entre $900 \mathrm{y}$ $1.050^{\circ} \mathrm{C}$ y preparación de pastillas a elevadas presiones, se podrían combinar los dos moles de $\mathrm{CaO}$. De lo anterior se deduce que el "compuesto $\mathrm{H}$ " tendría la siguiente fórmula:

$\mathrm{XCaO}_{2} \mathrm{YK}_{2} \mathrm{O} \cdot 1 / 2 \mathrm{CaF}_{2} \cdot 3\left(2 \mathrm{CaO} \mathrm{SiO}_{2}\right) \cdot(2-1) \mathrm{CaSO}_{4}$

\section{REACCIONES KF-CaSO ${ }_{4}-\mathrm{C}_{2} \mathrm{~S}$}

Con el fin de averiguar la influencia simultánea del $\mathrm{K}_{2} \mathrm{O}$ y del $\mathrm{CaSO}_{4}$ en la formación del "compuesto $\mathrm{H}$ " se hizo otra serie de ensayos, en los que se variaron las concentraciones molares de $\mathrm{K}_{2} \mathrm{O}$ a $1 / 3,1 / 2,2 / 3$ y 1 respectivamente y las del $\mathrm{CaSO}_{4}$ entre 1 y 2 moles; el $2 \mathrm{CaO} . \mathrm{SiO}_{2}$ se mantuvo constante en 3 moles.

* $\mathbf{C}=\mathrm{CaO} ; \mathbf{S}=\mathrm{SiO}_{2}$ según la nomenclatura de química del cemento. 
De los resultados obtenidos de los difractogramas se deduce que la cantidad óptima de $\mathrm{K}_{2} \mathrm{O}$ era de $1 / 2 \mathrm{~mol}$ que muy posiblemente se combinaba con el $\mathrm{CaF}_{2}$ según la reacción:

$$
1 / 2 \mathrm{~K}_{2} \mathrm{SO}_{4}+1 / 2 \mathrm{CaF}_{2} \Longleftrightarrow \mathrm{KF}+1 / 2 \mathrm{CaSO}_{4}
$$

El tiempo de tratamiento y un aumento de temperatura de 900 a $1.000^{\circ} \mathrm{C}$ favorecían la combinación. En el caso de $1 / 2 \mathrm{~mol}$ de $\mathrm{K}_{2} \mathrm{O}$ y $2 \mathrm{CaSO}_{4}$ el único componente potásico detectado era el "compuesto $\mathrm{H}$ ".

Si la cantidad de $\mathrm{K}_{2} \mathrm{O}$ era superior a $1 / 2 \mathrm{~mol}_{0}$ los difractogramas de las muestras presentaban las líneas correspondientes al $\mathrm{K}_{2} \mathrm{SO}_{4}$ y/o $\mathrm{K}_{2} \mathrm{SO}_{4} \cdot 2 \mathrm{CaSO}_{4}$.

Si la cantidad de $\mathrm{K}_{2} \mathrm{O}$ era igual o menor de $1 / 3$ de mol, parte de la fluorellestadita, que se formaba en este caso, permanecía sin descomponerse, mientras que en el caso de que el $\mathrm{K}_{2} \mathrm{O}$ fuese superior a $1 / 2 \mathrm{~mol}$, la fluorellestadita disminuía con el tiempo de tratamiento a $1.000^{\circ} \mathrm{C}$ y simultáneamente se formaba langbeinita.

De lo anterior se aceptó como fórmula para el "compuesto H".

$$
(2-1,6) \mathrm{CaO} \cdot \mathrm{KF} \cdot 3\left(2 \mathrm{CaO} \cdot \mathrm{SiO}_{2}\right) \cdot 2 \mathrm{CaSO}_{4}
$$

\subsection{Estudio de las temperaturas, del tiempo y de lasiconstantes cristalográficas del 2CaO.KF.3(2CaO.SiO $\left.{ }_{2}\right)$.2CaSO 4}

En la figura 4 se puede observar que a $800^{\circ} \mathrm{C}$ no se descomponía la calcita ni reaccionaban los componentes, cosa que sí sucedía al llegar la temperatura a $850^{\circ} \mathrm{C}$. Sin embargo para que se produzca el compuesto potásico de la fluorellestadita y ésta se descomponga, es preciso que la temperatura ascienda a $900^{\circ} \mathrm{C}$ y que la duración del tratamiento a dicha temperatura sea del orden de unas 50 horas.

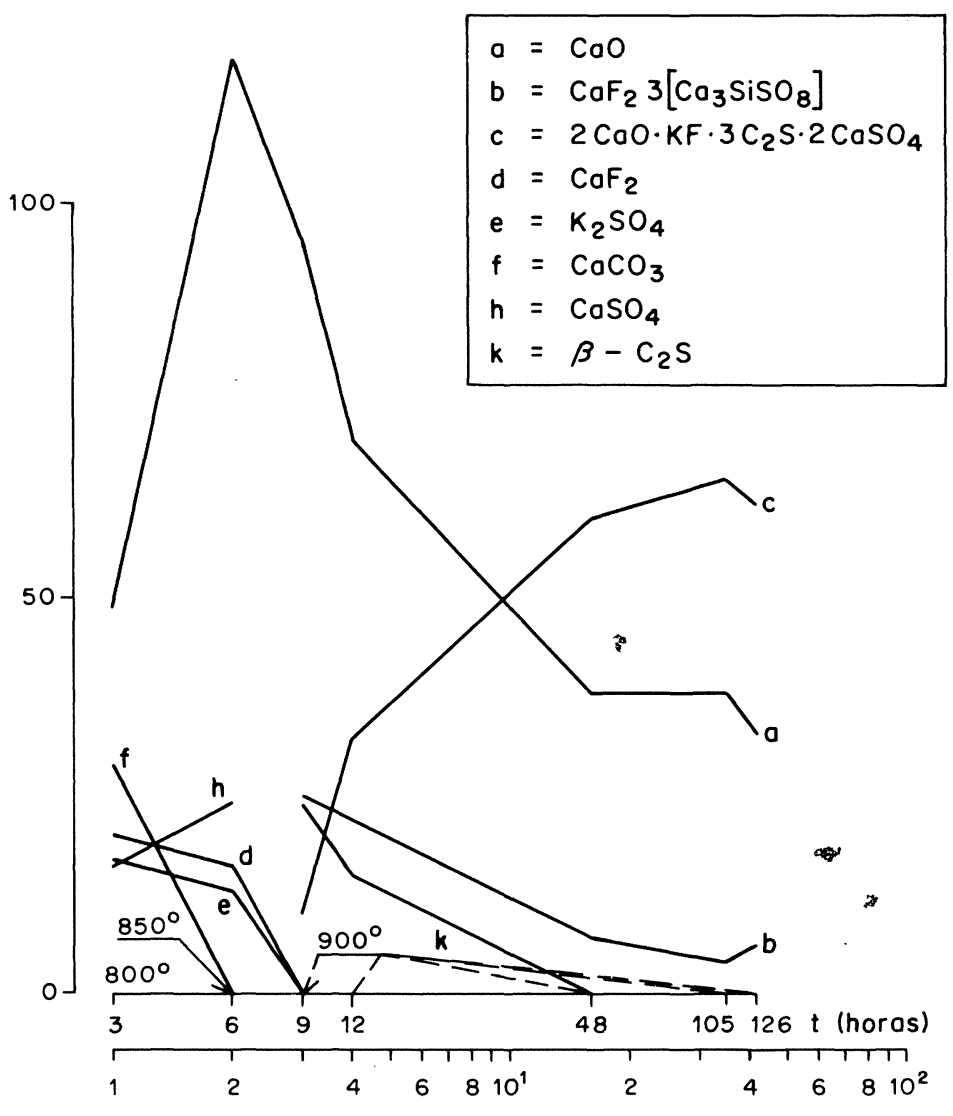

F1g. 4 
CUADRO 2

\begin{tabular}{|c|c|c|c|c|}
\hline n.o & $2 \theta$ & d & hkl & $\mathbf{I} / \mathbf{I}_{0}$ \\
\hline 1 & 6,433 & 13,7386 & 100 & 3 \\
\hline 2 & 12,887 & 6,8692 & 200 & 3 \\
\hline 3 & 14,374 & 6,1619 & 010 & 3 \\
\hline 4 & 17,872 & 4,9632 & 210 & 4 \\
\hline 5 & 24,745 & 3,5977 & 001 & 6 \\
\hline 6 & 25,594 & 3,4804 & 101 & 3 \\
\hline 7 & 25,886 & 3,4417 & 310 & 4 \\
\hline 8 & 28,732 & $3,1069^{\circ}$ & 011 & 10 \\
\hline 9 & 28,9906 & 3,0798 & 111 & 3 \\
\hline 10 & 30,6551 & 2,9163 & 120 & 1 \\
\hline 11 & 31,6290 & 2,8287 & 410 & 13 \\
\hline 12 & 32,5589 & 2,7500 & 320 & 100 \\
\hline 13 & 33,5794 & 2,6687 & 220 & 7 \\
\hline 14 & 36,1550 & 2,4843 & 401 & 4 \\
\hline 15 & 39,3484 & 2,2897 & 600 & 2 \\
\hline 16 & 39,7869 & 2,2655 & 121 & 0,05 \\
\hline 17 & 40,5067 & 2,22369 & 411 & 0,05 \\
\hline 18 & 41,3215 & 2,18485 & 321 & 2 \\
\hline 19 & 42,1771 & 2,14249 & 420 & 8 \\
\hline 20 & 44,0874 & 2,05399 & 030 & 0,2 \\
\hline 21 & 45,6712 & 1,98609 & 330 & 8 \\
\hline 22 & 46,4506 & 1,95485 & 710 & 0,5 \\
\hline 23 & 47,4558 & 1,91576 & 520 & 0,5 \\
\hline 24 & 48,0588 & 1,89312 & 230 & 4 \\
\hline 25 & 48,1663 & 1,88915 & 430 & 3 \\
\hline 26 & 50,7496 & 1,79888 & 002 & 15 \\
\hline 27 & 50,8542 & 1,79543 & 710 & 1 \\
\hline 28 & 51,353 & 1,77015 & 330 & 1 \\
\hline 29 & 53,25 & 1,72016 & 711 & 1 \\
\hline 30 & 57,80 & 1,59511 & 721 & 0,5 \\
\hline 31 & 59,60 & 1,55117 & 811 & . 2 \\
\hline 32 & 61,55 & 1,50662 & 140 & 6 \\
\hline
\end{tabular}

100 
La disminución de la cal libre se puede acelerar haciendo comprimidos o aumentando la temperatura a $1.000^{\circ} \mathrm{C}$ durante unas 3 horas.

En los difractogramas de la muestra no se detectó $\mathrm{CaF}_{2}$ lo cual confirmó nuestra anterior hipótesis; para eliminar el $\beta-2 \mathrm{CaO}^{-\mathrm{SiO}_{2}}$ y la fluorellestadita se necesitaron entre 1 y 2 moles de $\mathrm{CaO}$. $\mathrm{El}^{\mathrm{CaSO}_{4}}$ varía entre 1,5 y 2 moles.

Anteriormente se había calculado - a partir de las líneas de difracción del "compuesto H" en un programa (2) preparado en el Instituto Rocasolano con un M 20 (4) igual a 14- el volumen atómico del compuesto igual a 307,9 con una desviación estándar de 0,0001129.

Las densidades obtenidas con benceno oscilaban entre 2,88 y 3,12 , lo cual nos daba un peso molecular para el compuesto comprendido entre 887 y 960 ; esto nos indicaba como fórmulas más probables para el compuesto las siguientes:

$\begin{array}{lcc} & \text { p.m } & \text { d. } \\ \mathrm{KF} .2 \mathrm{CaO} . \mathrm{SiO}_{2} \cdot 4 \mathrm{CaSO}_{4} \cdot 2 \mathrm{CaO} & 887 & 2,88 \\ \mathrm{KF} .3\left(2 \mathrm{CaO} \cdot \mathrm{SiO}_{2}\right) \cdot 2 \mathrm{CaSO}_{4} \cdot \mathrm{CaO} & 903 & 2,97 \\ \mathrm{KF} .2\left(2 \mathrm{CaO} \cdot \mathrm{SiO}_{2}\right) \cdot 3 \mathrm{CaSO}_{4} \cdot 2 \mathrm{CaO} & 923 & 2,99 \\ \mathrm{KF} .2\left(2 \mathrm{CaO} . \mathrm{SiO}_{2}\right) \cdot 4 \mathrm{CaSO}_{4} & 947 & 3,07 \\ \mathrm{KF} .3\left(2 \mathrm{CaO} \cdot \mathrm{SiO}_{2}\right) \cdot 2 \mathrm{CaSO}_{4} \cdot 2 \mathrm{CaO} & 959 & 3,11\end{array}$

La densidad medida con agua fue de 3,1 . El compuesto KF.3 $\left(2 \mathrm{CaO}_{\mathrm{SiO}}\right) \cdot 2 \mathrm{CaSO}_{4} \cdot \mathrm{CaO}$ admitía más cantidad de $\mathrm{CaO}$, por lo cual creemos que la fórmula más adecuada para el "compuesto $\mathrm{H}$ " denominado ahora potasio fluorellestadita es:

$$
\mathrm{KF} .3\left(2 \mathrm{CaO} . \mathrm{SiO}_{2}\right) \cdot 2 \mathrm{CaSO}_{4} \cdot 2 \mathrm{CaO}
$$

Las constantes cristalográficas, líneas de difracción, índices e intensidades del compuesto se dan en el cuadro 2 y en los datos que se indican a continuación:

$$
\begin{array}{llll}
\text { celda monoclínica; } & \mathrm{A}=13,88 ; & \mathrm{B}=6,29 ; & \mathrm{C}=3,597 \\
\alpha=90^{\circ} & \beta=90^{\circ} & \gamma=98,26^{\circ}
\end{array}
$$

\section{B I B L I O G R A F I A}

(1) Inorganic Index to the Powder Diffraction Fille of the JCPDS (1972).

(2) Indexing Program for Powder Patterns Especially Suitable for Triclinic Monoclinic and Orthorhombic Lattices.

F. Kohlbeck y E. M. Horl J. Appl. Cryst. 928 (1976).

(3) Sudoh y Nakamura: Review of Twenty Fifth General Meeting, 84 (1971).

(4) A Simplified Criterion for the Reeliability of a Powder Pattern Indexing. P. M. de Wolff. J. Appl. Cryst. 1, 108 (1968).

(5) Takashima, S.: Semento Gijutzu Nempo XII, pg. 12-13 (1958). 